\title{
Prompt neutron multiplicity in correlation with fragments from spontaneous fission of ${ }^{252} \mathrm{Cf}$
}

\author{
A. Göök, F.-J. Hambsch, ${ }^{*}$ and M. Vidali \\ European Commission, Joint Research Centre, Institute for Reference Materials and Measurements, Retieseweg 111, B-2440, Geel, Belgium
}

(Received 23 September 2014; published 12 December 2014)

\begin{abstract}
The spontaneous fission of ${ }^{252} \mathrm{Cf}$ serves as an excellent benchmark of prompt emission in fission since experimental data can be obtained without the need of an incident beam. With the purpose of providing experimental data on the prompt fission neutron properties in correlation with fission-fragment characteristics, an experiment on ${ }^{252} \mathrm{Cf}(\mathrm{SF})$ has been performed. In addition, the experiment serves as a benchmark of setup and analysis procedures for measurements of fluctuations in the prompt-neutron properties as a function of incident neutron energy in fission of the major actinides ${ }^{235} \mathrm{U}$ and ${ }^{239} \mathrm{Pu}$. The experiment employs a twin Frisch grid ionization chamber as fission-fragment detector while neutrons were counted by using a liquid scintillator placed along the symmetry axis of the ionization chamber. Average neutron multiplicity has been obtained as a function of fission-fragment mass and total kinetic energy (TKE). The average multiplicity as a function of mass agrees well with available data in the literature in the mass range from 80 to $170 \mathrm{u}$. The existence of additional sawtooth structures in the far asymmetric mass region could not be confirmed, although the statistical accuracy of the present experiment is as good as the previous study where such structures have been reported [Nucl. Phys. A 490, 307 (1988).]. The available data in the literature on the TKE dependence of the multiplicity show strong deviations. Therefore, effort was focused on investigating experimental factors in low-efficiency neutron-counting experiments that may lead to faulty determination of this dependence. Taking these factors into account, a result that agrees well with data from high-efficiency neutron-counting experiments is obtained. The experimental arrangement allows determination of the angle between the detected neutron and the fission axis, which permits the neutron properties to be transformed into the fission-fragment rest frame. Fission neutron emission spectra in the fragment center-of-mass frame have thereby been obtained as a function of the fission-fragment mass and TKE.
\end{abstract}

DOI: 10.1103/PhysRevC.90.064611

PACS number(s): 28.20.-v, 24.75.+i, 25.85.Ca

\section{INTRODUCTION}

Investigation of prompt-fission neutron emission in fission is of particular importance in understanding the fission process. Knowledge of the properties of prompt-fission neutrons, their multiplicities, and energy distributions could give answers to questions related not only to neutron emission itself but also to questions relevant to the formation of the fission fragments, the sharing of excitation energy among them, and the timescale of the process. For nuclear modeling and improved evaluation of nuclear data, the knowledge of fluctuations in the promptneutron multiplicity as a function of incident neutron energy is requested for the major actinides ${ }^{235} \mathrm{U}$ and ${ }^{239} \mathrm{Pu}$. Fluctuations in fission-fragment mass and total kinetic energy (TKE) in both isotopes have been observed in resonance neutron-induced fission [1,2]. Independently, fluctuations in the number of emitted neutrons have also been observed [3]. In view of the fact that both neutron number and fission-fragment properties have been found to vary, it is necessary to understand to what extent the prompt neutron multiplicity is changing or if the observed fluctuations are due to the changes in the fission-fragment properties. Furthermore, knowledge of the

\section{*Franz-Josef.HAMBSCH@ec.europa.eu}

Published by the American Physical Society under the terms of the Creative Commons Attribution 3.0 License. Further distribution of this work must maintain attribution to the author(s) and the published article's title, journal citation, and DOI. prompt neutron multiplicity as a function of mass and TKE is needed when determining post-neutron-emission fissionfragment mass distributions experimentally via the double kinetic energy or double velocity techniques. In Ref. [4], the impact on mass distributions by fluctuations of the mass dependence of the neutron multiplicity as a function of incident neutron energy was investigated. In the most severe cases the relative impact can be as large as $20 \%-30 \%$.

Experimental investigations of the correlations of promptfission neutron multiplicity with fragment properties in resonance neutron-induced fission of ${ }^{235} \mathrm{U}$ and ${ }^{239} \mathrm{Pu}$ are taking place at the Geel Electron Linear Accelerator (GELINA) facility of the Joint Research Centre, Institute for Reference Materials and Measurements (JRC-IRMM), employing an array of neutron scintillation detectors (SCINTIA). Here, an experimental investigation of prompt-fission neutron multiplicity correlations with fission-fragment mass and energy in the spontaneous fission of ${ }^{252} \mathrm{Cf}$ is presented. The experiments were undertaken to verify the experimental setup and analysis procedures relevant for implementation of the SCINTIA neutron detector array. Emphasis was put on understanding the behavior of the total number of neutrons emitted as a function of total kinetic energy, $\bar{v}_{T}(\mathrm{TKE})$, where discrepancies have recently been identified [5-7]. In addition, a fully digital data acquisition system and digital signal processing was implemented. Beside the purely experimental interest, recent advances in the theoretical description of prompt emission in fission [8-10] require detailed data for verifying the models. The ${ }^{252} \mathrm{Cf}(\mathrm{SF})$ decay serve also as an excellent benchmark of these calculations. 

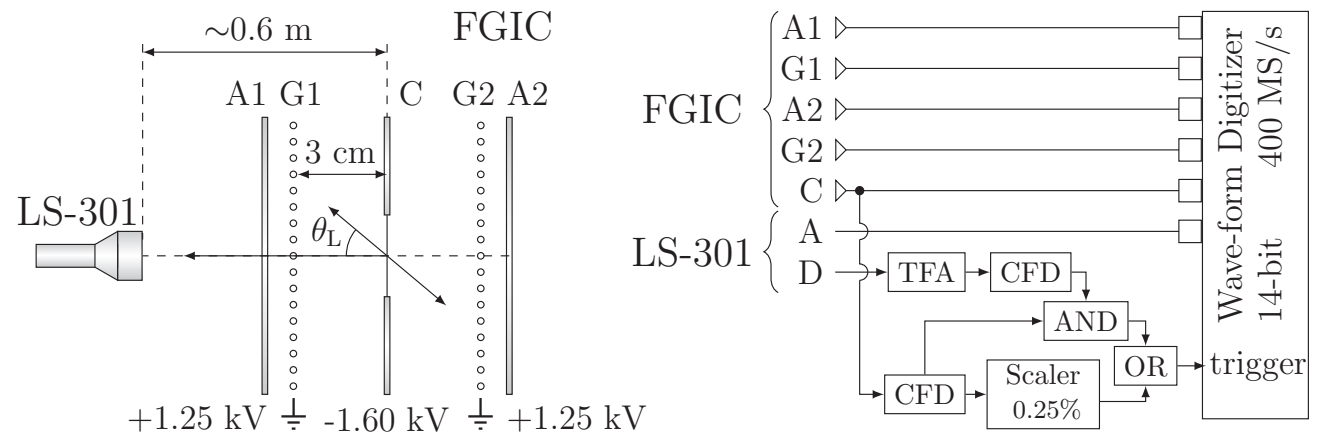

FIG. 1. Schematic drawing of the experimental setup (left) with the NE-213-type neutron detector (LS-301) and the twin-Frisch grid ionization chamber, as well as the associated electronic setup (right). The abbreviations are CFD for constant fraction discriminator, and TFA for timing filter amplifier.

\section{EXPERIMENTAL METHOD}

The experimental setup was similar to that of the experiment performed at JRC-IRMM (formerly the Central Bureau for Nuclear Measurements, or CBNM) by Budtz-Jørgensen and Knitter [11] in 1988. A schematic drawing is shown in Fig. 1. An NE-213-type liquid scintillator $(127 \mathrm{~mm} \times 51 \mathrm{~mm})$ was placed along the symmetry axis of a twin Frish-grid ionization chamber (FGIC). A thin layer of ${ }^{252} \mathrm{Cf}$ (716 fissions/s) on a thin Ni backing $\left(220 \mu \mathrm{g} / \mathrm{cm}^{2}\right)$, which allows simultaneous observation of both fission fragments, was placed in a hole in the central common cathode. The distance between the target and the center of the scintillator volume was $59.95 \mathrm{~cm}$. As counting gas, a P-10 gas mixture (90\% Ar $+10 \%$ $\mathrm{CH}_{4}$ ) was utilized, flowing through the FGIC at a rate of $\sim 50 \mathrm{ml} / \mathrm{min}$ and kept at a pressure of $1.085 \times 10^{5} \mathrm{~Pa}$. The grids were of the crossed-wire-mesh type, with a pitch of $480 \mu \mathrm{m}$ and wire radius of $28 \mu \mathrm{m}$. The distance between the anode and grid was $6 \mathrm{~mm}$, while the distance between cathode and grid was $31 \mathrm{~mm}$. The ionization chamber signals were amplified by using current-sensitive preamplifiers. The output of the preamplifiers as well as the neutron detector photomultiplier anode were directly digitized in a 14-bit, $400 \mathrm{Msample} / \mathrm{s}$ waveform digitizer and stored on disk for further offline treatment. A coincidence between the cathode signal and that from one of the neutron detector dynodes was used to trigger the acquisition. In addition, triggers from the ionization chamber without requiring coincidences with the neutron detector were accepted at a rate scaled down to $0.25 \%$. This allowed for monitoring of electronic stability during the measurement by a continuous evaluation of the noncoincident fission-fragment distribution, without a large increase in dead-time of the acquisition system that would be present if all fission events were accepted continuously.

\section{DATA TREATMENT}

\section{A. Digital-signal processing}

The treatment of the digitized waveforms from the ionization chamber essentially follows the procedures outlined in Ref. [12], including correction for pileups on the trailing edge of the signals. Two major differences were implemented: a leading-edge pileup rejection scheme and determination of the fission-fragment emission angle from the drift time of ionization electrons.

For a single event in the ionization chamber, the width of the cathode current signal is theoretically the same for all events; namely, the time it takes electrons created at the cathode to drift to the grid. Because of this fact the width of the registered signal can be used to identify pileup events. In practice this is done by counting the number of samples above the electronic noise level of the digitized cathode signal waveform. The fall time of the cathode-current signal depends on the orientation of the fission-fragment track relative to the ionization chamber axis. Because of this the determined width will also have a dependence on the orientation of the track. The time over threshold is therefore written in the output file of the digital waveform analysis for each event, so that the cutoff value can be made as a function of the cosine of the emission angle, once it has been determined. In Fig. 2 the cathode-current signal waveform from three different types of events is shown, as identified by the pileup identification algorithm. Represented by the black line is a clean fission event, while blue and red lines represent $\alpha$-particle and fission-fragment pileup, respectively. In Fig. 3 the result for the anode pulse-height spectrum and the neutron time-of-flight spectrum of applying the pileup rejection scheme is illustrated;

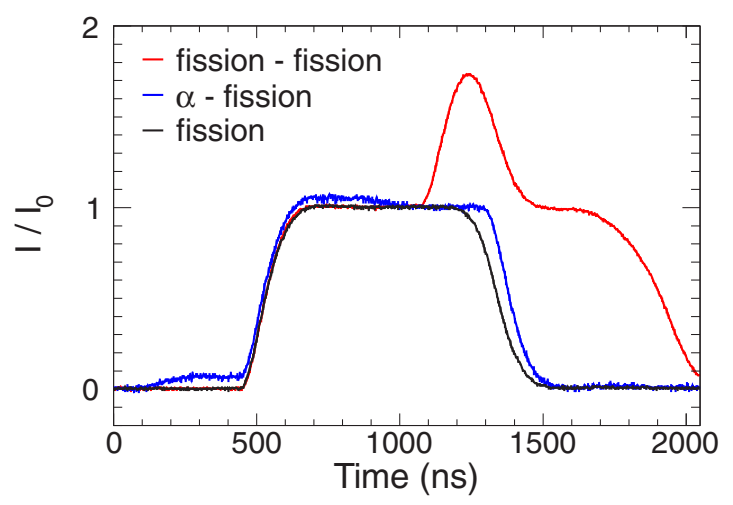

FIG. 2. (Color online) Cathode-current signal wave forms for a clean fission event (black line), an event suffering from an $\alpha$-particle pileup (blue line), and an event suffering from a fission pileup (red line), as identified by the pileup identification algorithm. 

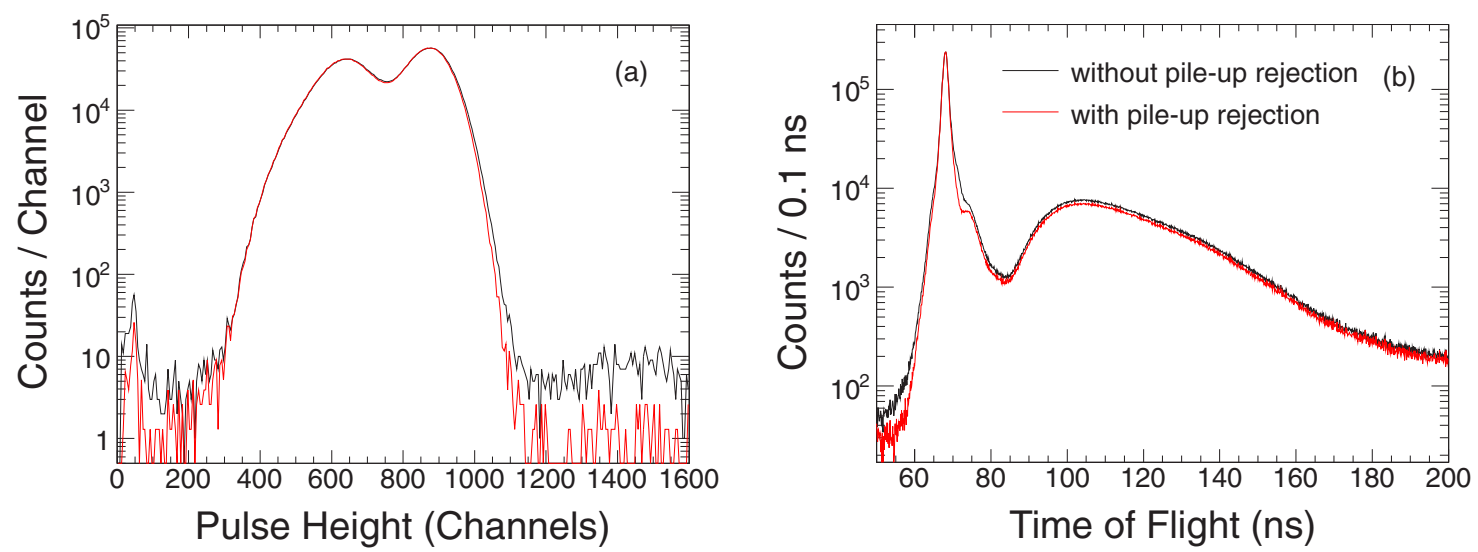

FIG. 3. (Color online) (a) Anode-pulse-height distribution before (black line) and after (red line) pileup rejection; the latter has been scaled to coincide with the former at the light fragment peak. (b) Time-of-flight spectrum before (black line) and after (red line) pileup rejection; the latter has been scaled to coincide with the former at the prompt- $\gamma$ peak.

these data were taken with a ${ }^{252} \mathrm{Cf}$ source with an activity of $3.28 \times 10^{3}$ fission/s. The main effect of the pileup rejection on the anode pulse-height distribution is a decrease in events to the right of the double-humped fission-fragment distribution, a slight increase in the peak-to-valley ratio is also observed. With pileup rejection in the ionization chamber, a slight reduction in the width of the prompt- $\gamma$-ray peak is observed in the time-of-flight spectrum.

The cosine of the fission fragment's emission angle relative to the electrode normal was derived from the average drift time of ionization electrons. The drift time was determined by analyzing the digitized pulse shape of the ionization chamber anode signals by using the following relation:

$$
\bar{t}_{d}=\frac{\sum_{k=k_{0}}^{k_{0}+\Delta k} i_{k} k}{\sum_{k=k_{0}}^{k_{0}+\Delta k} i_{k}} \delta t-t_{0},
$$

where $k$ is the sample number, $i$ is the amplitude of the digitized anode-current signal, $t_{0}$ is the moment of fission, $k_{0}$ is the corresponding sample number, $\Delta k$ is the length of the time window in terms of the number of samples, and $\delta t$ is the sampling interval. The cosine of the emission angle is given by

$$
\cos \theta=\frac{\bar{t}_{d}\left(90^{\circ}\right)-\bar{t}_{d}(\theta)}{\bar{t}_{d}\left(90^{\circ}\right)-\bar{t}_{d}\left(0^{\circ}\right)} .
$$

The procedure to find $\cos \theta$ is similar to the standard procedure using the pulse height from the grid electrode or sum signal (see, for example, Ref. [12]). In Fig. 4(a) the angular distribution of fission fragments (when not requiring coincidence with the neutron detector) determined from the drift time is shown: the distribution is clearly isotropic with a decrease in yield at $\cos \theta \lesssim 0.3$ due to target absorption and scattering effects. Figure 4(b) displays the distribution of the difference in $\cos \theta$ determined for the two chamber sides. The resolution in the value of $\cos \theta$ of the fission axis (taken as the average of the values determined from the two chamber sides) is given by the half width of the peak, which amounts to $4.4 \%$.

\section{B. Pulse-shape discrimination}

$\gamma$-ray background in the scintillator was suppressed by using digital pulse-shape-discrimination techniques. The best background suppression was achieved by using a combination of two different pulse-shape-discrimination techniques: one based on sensing the fall time of the pulse (zero-crossing
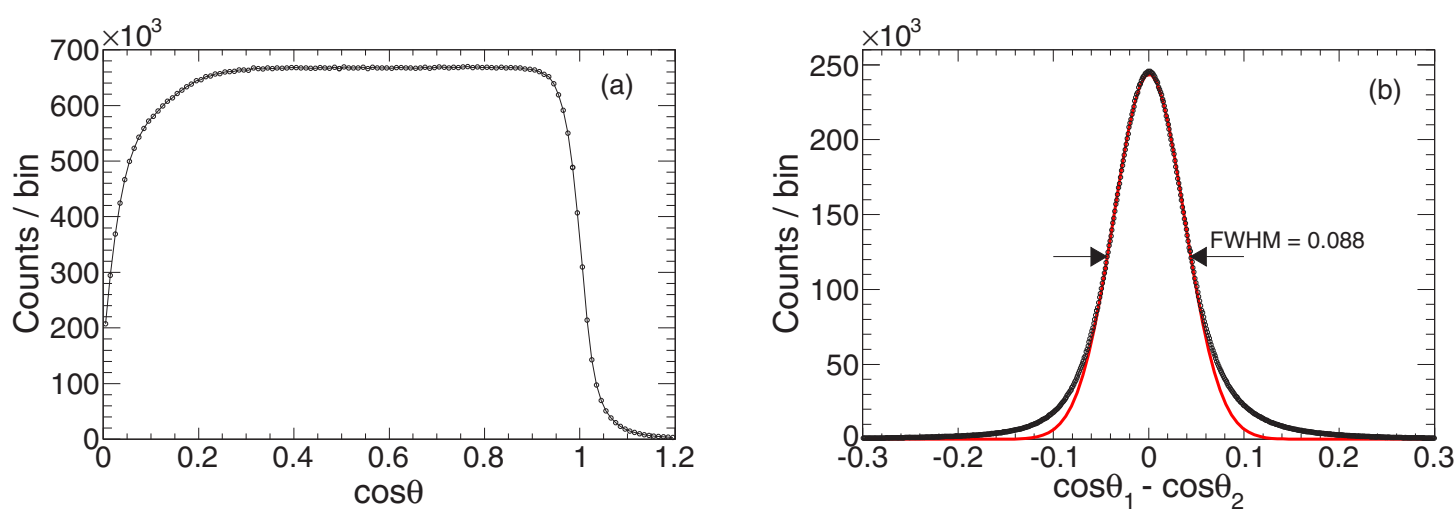

FIG. 4. (Color online) (a) Distribution of fission fragments as a function of the detected $\cos \theta$ value. (b) Distribution of the difference in $\cos \theta$ values detected for fragments on the sample and backing side, together with a Gaussian fit. 

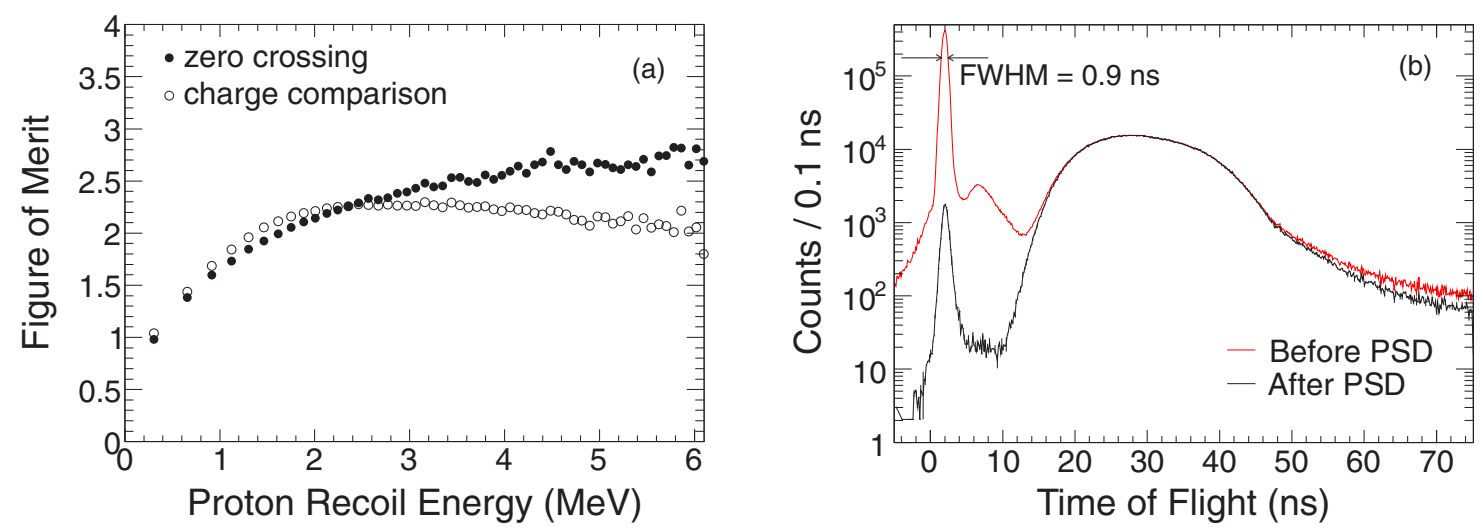

FIG. 5. (Color online) (a) Comparison of the figure of merit of $n-\gamma$ separation as a function of the proton recoil energy calibrated pulse height for the two methods of pulse-shape discrimination. (b) Time-of-flight spectrum before and after PSD.

method) and another based on integrating the pulse in two different regions (charge-comparison method). To sense the fall time of the pulse a differentiation-double-integration $\left(\mathrm{CR}-\mathrm{RC}^{2}\right.$ ) shaping algorithm was used. The CR-RC $\mathrm{C}^{2}$ algorithm produces a bipolar pulse whose zero-crossing time is dependent of the fall time of the pulse. In the charge comparison method the pulse is integrated in two different regions: a full and a delayed region. The ratio of the two integrals contains the particle-type information. The quality of $n-\gamma$ separation can be quantitatively compared by using the figure of merit, defined according to

$$
M=\frac{x_{\gamma}-x_{n}}{w_{\gamma}+w_{n}}
$$

where $x_{\gamma}$ and $x_{n}$ are the centers of the $\gamma$-ray and neutron peaks, respectively, and $w_{\gamma}, w_{n}$ are the corresponding peaks' full width at half maximum (FWHM). In Fig. 5(a) the figure of merit for the two methods is compared; it is clear that the two methods are comparable in accuracy but also that neither of them is better than the other over the entire pulse-height range. Hence, a combination of both methods was used in the analysis. At low pulse heights the pulse-shape discrimination fails for both methods; therefore, a pulse-height threshold corresponding to $0.7 \mathrm{MeV}$ proton-recoil energy $(\sim 100 \mathrm{keVee})$ was applied. The final result for the neutron time-of-flight spectrum is shown in Fig. 5(b), the prompt- $\gamma$-ray peak is reduced by more than two orders of magnitude by pulse-shape discrimination. The bump around $6 \mathrm{~ns}$ in the time-of-flight spectrum, which can be identified as $\gamma$ rays from inelastic scattering from the ionization chamber, is also greatly reduced.

\section{Neutron-energy determination and detection efficiency}

The neutron energy in the laboratory system was determined via the conventional time-of-flight technique; the flight time is given by the difference between the time pick off from the neutron detector and the ionization chamber cathode signals. Digital shaping algorithms and an algorithm emulating the electronic shaping steps of analog constant fraction discriminators were used for the time pick off of the detector signals, giving a time-of-flight resolution of $0.9 \mathrm{~ns}$
FWHM. The absolute time offset was determined from the position of the prompt-fission gamma-ray peak.

The neutron detector efficiency as a function of the neutron energy was determined from the ratio of the observed energy spectrum and the Mannhart evaluation [13]. The pulse-height scale was calibrated in terms of the recoil-proton energy according to the procedure from Ref. [14].

\section{Derivation of fission-fragment masses and energies}

The knowledge of the fission-fragment emission angle relative to the chamber axis is imperative for correct determination of the fission-fragment energies. This knowledge is used to correct for angle-dependent systematic errors in the pulse heights due to grid inefficiency [16] and energy loss in target and backing materials [17]. Once these systematic errors have been corrected for, the iterative procedure for calculating pre- and post-neutron energies and masses can begin. The first iterative procedure starts by calculating provisional mass numbers $\mu$ from the apparent energies $\varepsilon$ :

$$
\mu_{1,2}=252\left(\frac{\varepsilon_{2,1}}{\varepsilon_{1}+\varepsilon_{2}}\right),
$$

where the index indicates the chamber side. By using the provisional mass number, the first approximation of the postneutron energy is calculated:

$$
E=\varepsilon+\Delta(\mu, \varepsilon),
$$

where $\Delta(\mu, \varepsilon)$ is the pulse-height defect, which was taken from Ref. [18]. From $E$ a new approximation of the provisional mass is calculated. The iterative procedure continues as long as the mass number from Eq. (4) differs by more than 0.15 mass units from the previous iteration. Once the entire data set is analyzed a first approximation of the neutron multiplicity distribution $\bar{v}(\mu$, TKE) can be derived, see Sec. III E. From the post-neutron energy and provisional mass the pre-neutron energy can then be calculated:

$$
E^{*}=E \frac{\mu}{\mu-\bar{v}(\mu, \mathrm{TKE})},
$$

where TKE $=E_{1}+E_{2}$ is the post-neutron total kinetic energy. The second iterative procedure starts from Eq. (4) replacing 

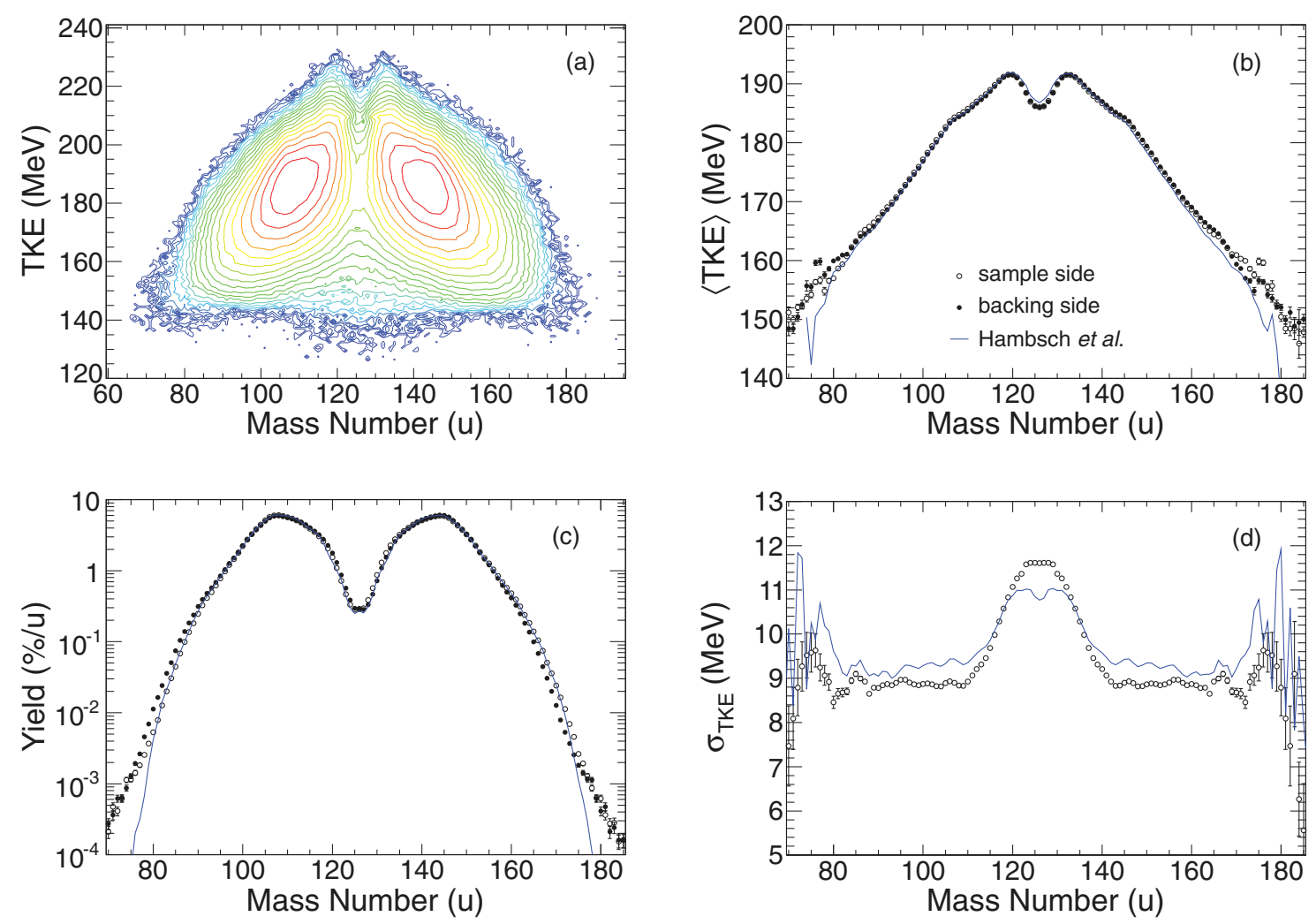

FIG. 6. (Color online) (a) Fission-fragment yield as a function of pre-neutron mass and TKE, (b) pre-neutron-mass distribution, (c) average TKE, and (d) standard deviation of the TKE as a function of the fission-fragment mass number from this work, compared with data from Ref. [15].

$\varepsilon$ by $E^{*}$ in Eq. (4) and by $E$ when calculating the pulseheight defect in Eq. (5). In this way the provisional mass $\mu$ converges to the pre-neutron mass number $m^{*}$. In Fig. 6 the fission-fragment yield determined as a function of mass and TKE is shown; also shown are the projection on the mass axis and the average as well as the standard deviation of the $\mathrm{TKE}$ as a function of mass in comparison with literature data [15]. Reasonable agreement of the fission-fragment properties is observed. The apparent increase in yield for far asymmetric mass splits is an artifact induced by the large solid angle of acceptance $(\cos \theta \geqslant 0.5)$ in this experiment, which causes multiple scattered fragments to play a more important role (see Ref. [15] for details).

\section{E. Determination of prompt-fission-neutron multiplicities}

The average number of neutrons emitted by a fragment of mass $m^{*}$ from a fission of total kinetic energy TKE* is given by

$$
\bar{v}\left(m^{*}, \mathrm{TKE}^{*}\right)=\frac{1}{N_{F}\left(m^{*}, \mathrm{TKE}^{*}\right)} \sum_{E_{L}} \frac{N_{C}\left(m^{*}, \mathrm{TKE}^{*}, E_{L}\right)}{\varepsilon_{T}\left(E_{L}\right)},
$$

where $N_{F}$ is the total number of fissions, $N_{C}$ is the number of fission-neutron coincidences, $E_{L}$ is the neutron energy in the laboratory frame, and $\varepsilon_{T}$ is the total neutron detection efficiency. Besides the neutrons detected from the fragment of mass $m^{*}$ it is also possible to detect neutrons originating from the fragments flying in the opposite direction. However, the contribution of this type of event is small and may be treated as a small perturbation [11].

A vector diagram of the kinematics of neutron emission from a fully accelerated fragment is depicted in Fig. 7. Transformation from the laboratory frame to the fragment center-of-mass frame for neutrons emitted by the fragment flying into the hemisphere of the neutron detector can be written as

$$
\begin{aligned}
v_{\text {c.m. }}^{2} & =v_{\mathrm{L}}^{2}+v_{\mathrm{F}}^{2}-2 v_{\mathrm{L}} v_{\mathrm{F}} \cos \theta_{\mathrm{L}}, \\
\cos \theta_{\text {c.m. }} & =\frac{v_{\mathrm{L}} \cos \theta_{\mathrm{L}}-v_{\mathrm{F}}}{v_{\text {c.m. }}},
\end{aligned}
$$

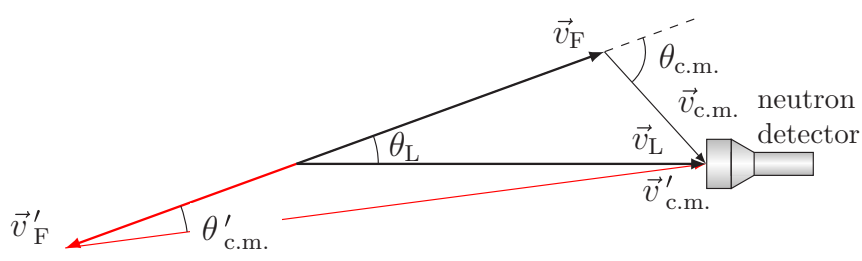

FIG. 7. (Color online) Vector diagram of the kinematics of neutron emission from a fully accelerated fragment and the transformation into the fragment rest frame (c.m.), black lines represent the fragment detected in the same hemisphere as the neutron, while red lines represent the complimentary fragment. The label for fragment velocity is $\vec{v}_{\mathrm{F}}$, for complimentary fragment velocity it is $\vec{v}_{\mathrm{F}}^{\prime}$, for neutron velocity in laboratory frame it is $\vec{v}_{\mathrm{L}}$, and for neutron velocity in the center-of-mass frame it is $\vec{v}_{\text {c.m. }}$. 


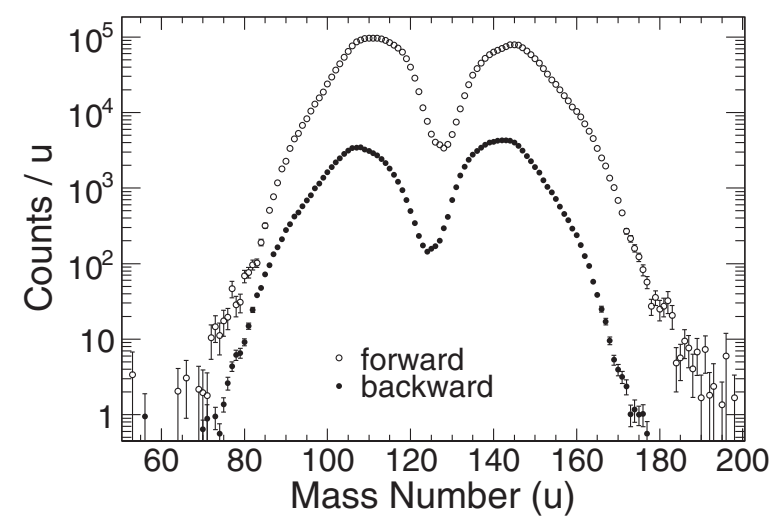

FIG. 8. Calculated contribution of neutron coincidences from fragments directed away from the neutron detector (closed points) compared with the recorded number of coincidences (open points) as a function of fragment mass number.

where all quantities are defined in Fig. 7. Only events with a center-of-mass emission angle $\theta_{\text {c.m. }} \geqslant 0^{\circ}$ are taken into account. This implies

$$
v_{\mathrm{L}} \geqslant \frac{v_{\mathrm{F}}}{\cos \theta_{\mathrm{L}}}
$$

i.e., only neutrons with a kinetic energy greater than the kinetic energy per nucleon of the corresponding fission fragment are included in the analysis. This reduces the importance of the low-energy part of the neutron spectrum, where scattered neutrons play a more important role. A first approximation of the neutron-multiplicity matrix $\bar{v}(\mu, \mathrm{TKE})$ is determined by using Eq. (7), without taking the neutron emission by the fragments into account when calculating the masses. This multiplicity matrix is then used in the second iterative calculation of the fission-fragment pre-neutron masses and energies; cf. Sec. III D. The basic assumption when correcting the fission-fragment energies for prompt-fission-neutron multiplicity in Eq. (6) is that neutrons are emitted isotropically in the fragment rest frame. Even if this were true (on average) for the set containing all fission events, it is not true for the subset of events where a neutron is also registered in the scintillator [19]. When determining mass and TKE for the coincident events, Eq. (6) must therefore be modified to take the known momentum transfer of the registered neutron into account:

$$
E^{*}=E \frac{\mu}{\mu-\bar{v}(\mu, \mathrm{TKE})}+2 \frac{E^{*}}{\mu}\left(\sqrt{E_{L} \frac{\mu}{E^{*}}} \cos \theta_{L}-1\right) .
$$

A distribution $N^{\prime}\left(m^{*}, \mathrm{TKE}^{*}\right)$ is generated by the event by event analysis. This distribution consist of coincidences from both the fragment flying in the forward and backward directions (towards and away from the neutron detector, respectively) and may be written as

$$
N^{\prime}\left(m_{f}^{*}, \mathrm{TKE}^{*}\right)=\epsilon_{f} N\left(m_{f}^{*}, \mathrm{TKE}^{*}\right)+\epsilon_{b} N\left(m_{b}^{*}, \mathrm{TKE}^{*}\right),
$$

where $N\left(m^{*}, \mathrm{TKE}^{*}\right)$ is the true number of neutrons emitted by a fragment with mass $m^{*}$ in a fission with total kinetic energy

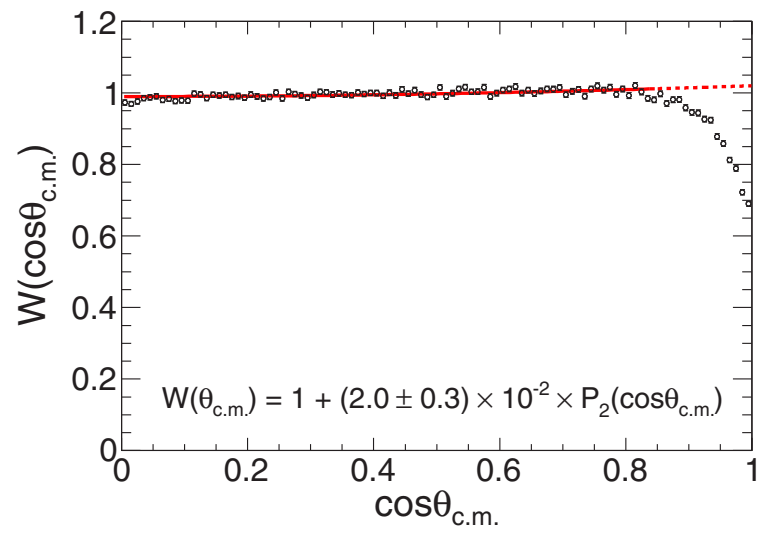

FIG. 9. (Color online) Prompt-fission-neutron angular distribution in the rest frame of the fission fragments. The red line represents the best fit of a second-order Legendre polynomial to the range $\cos \theta_{\text {c.m. }} \in[0,0.85]$.

$\mathrm{TKE}^{*}, m_{f}^{*}$ and $m_{b}^{*}$ are the masses of fragments detected in the forward and backward directions, respectively, while $\epsilon_{f}$ and $\epsilon_{b}$ are the probabilities of detecting the neutrons from these fragments. Since the probability of detecting a neutron from the fragment flying in the backward direction is comparatively small, we make the first-order approximation

$$
N\left(m^{*}, \mathrm{TKE}^{*}\right) \approx N^{\prime}\left(m^{*}, \mathrm{TKE}^{*}\right) .
$$

Replacing the second term in Eq. (12) by this expression, we obtain

$$
N\left(m_{f}^{*}, \mathrm{TKE}^{*}\right)=\frac{N^{\prime}\left(m_{f}^{*}, \mathrm{TKE}^{*}\right)-\epsilon_{b} N^{\prime}\left(m_{b}^{*}, \mathrm{TKE}^{*}\right)}{\epsilon_{f}} .
$$

The forward $\epsilon_{f}$ and backward $\epsilon_{b}$ efficiencies are found from the center-of-mass neutron-energy spectrum. For a given neutron energy in the laboratory frame, the center-of-mass energy for a neutron originating from the backward-directed fragment is substantially higher than for the neutron from the forward-directed fragment (cf. Fig. 7). By fitting the center-ofmass energy spectrum with the expression for cascade neutron

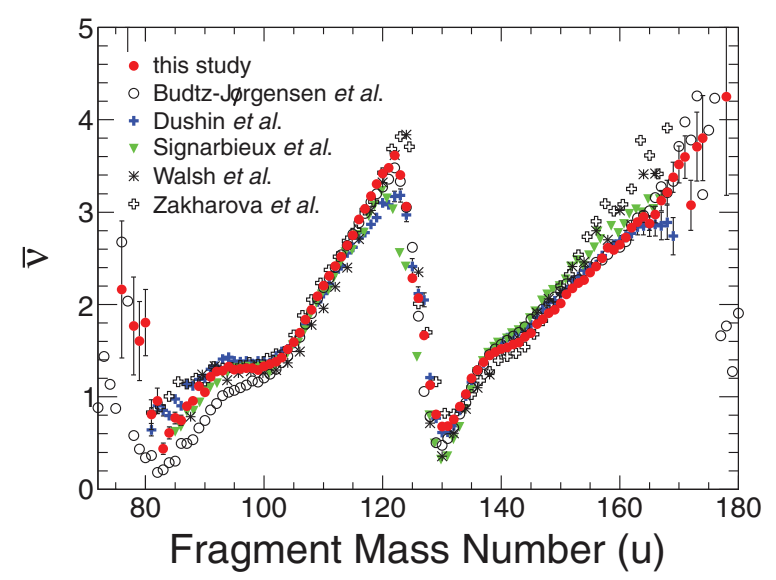

FIG. 10. (Color online) Average prompt-fission neutron multiplicity as a function of fission-fragment mass, in comparison with data from Refs. [11,22-25]. 


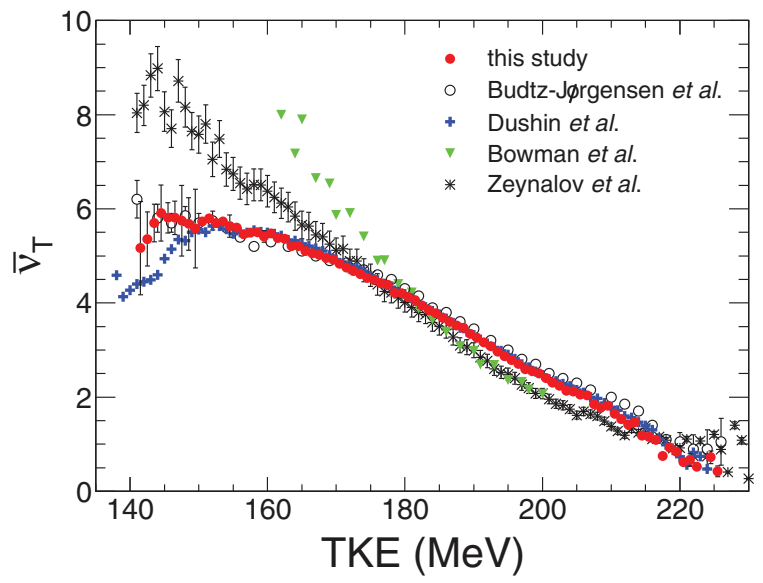

FIG. 11. (Color online) Total average prompt-fission neutron multiplicity as a function of TKE, in comparison with experimental data from Refs. [6,11,22,26].

emission [20],

$$
\Phi(\eta) \propto \eta^{\lambda} \exp \left\{-\eta / T_{\mathrm{eff}}\right\}
$$

the relative probability of neutrons originating from the forward- and backward-directed fragments can be obtained, where $\eta$ is the center-of-mass neutron energy while $\lambda$ and $T_{\text {eff }}$ are fit parameters. In Fig. 8 the calculated number of promptfission neutron coincidences from the complimentary fragment is compared with the number of recorded coincidences as a function of the coincident fragment mass in the forward and backward directions. Coincidences from the fragments in the backward direction is for most masses only a few percent; however, around $130 \mathrm{u}$ where the neutron multiplicity has a minimum the correction is larger than $10 \%$.

\section{EXPERIMENTAL RESULTS}

\section{A. Prompt-fission-neutron angular distributions}

By using Eq. (8) the fission neutron angular distributions obtained in the laboratory frame can be transformed into the rest frame of the fission fragments. Figure 9 displays this

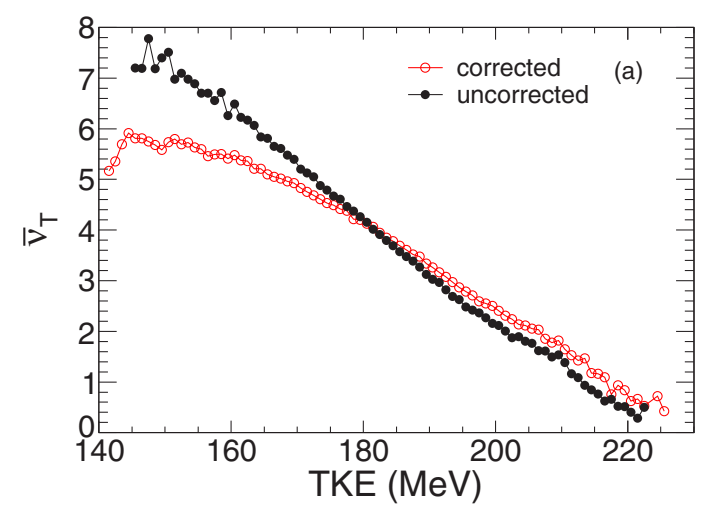

center-of-mass angular distribution fitted with a second-order Legendre polynomial

$$
W\left(\cos \theta_{\text {c.m. }}\right)=1+\alpha P_{2}\left(\cos \theta_{\text {c.m. }}\right),
$$

normalized such that

$$
\int_{0}^{1} W\left(\cos \theta_{\text {c.m. }}\right) d\left(\cos \theta_{\text {c.m. }}\right)=1 .
$$

The angular distribution is very close to isotropic. In fact, the amplitude of $P_{2}$ is, within statistical uncertainty, the same as in Ref. [11], where it was concluded that the angular distribution is isotropic. However, the statistical accuracy of the polynomial fit in this study suggests a nonisotropic component. In order to validate such a conclusion, further investigation of the systematic uncertainties associated with the determination of the center-of-mass angular distribution is needed; this will be the subject of another publication. We may, however, conclude that, if such an anisotropy truly exists, its magnitude is small and the assumption of isotropy in the fragment rest frame when treating the data will not have a large influence on the final result.

\section{B. Average prompt-fission-neutron multiplicities}

Because the efficiency of the neutron detector was extracted from the ratio of the experimental energy spectrum and the Mannhart evaluation, no result for the absolute value of the mean total multiplicity could be extracted. Instead, the multiplicity matrix $\bar{v}\left(m^{*}, \mathrm{TKE}\right)$ was normalized to $\bar{v}_{T}=3.759$ from the Evaluated Nuclear Data File (ENDF) library [21]. In total $\sim 3 \times 10^{6}$ prompt-neutron and fission-fragment coincidences survived the condition of Eq. (10) and were consequently used for the evaluation of the average prompt-neutron multiplicities.

The mass dependence of the prompt-fission-neutron multiplicity from this experiment is compared to data from the literature [11,22-25] in Fig. 10. The data from this study agree quite well with experimental data available in the literature. Close to the peak around mass number 120, large discrepancies in the available data are observed. This mass region is very sensitive to mass-resolution effects and correct treatment of the recoil energy imparted to the fission fragment by the detected neutron [19]. The enhanced multiplicity in the mass

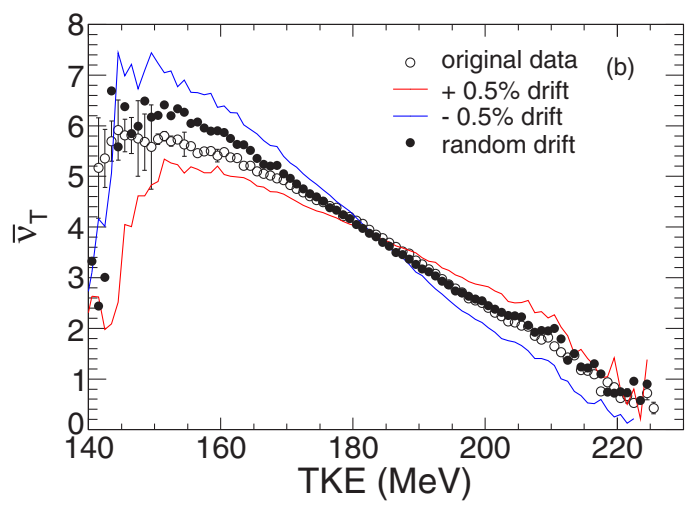

FIG. 12. (Color online) (a) Influence of neutron recoil correction on the average total prompt-fission-neutron multiplicity as a function of TKE in the present experiment. (b) Influence of electronic temperature drift on the average total prompt-fission-neutron multiplicity as a function of TKE. 

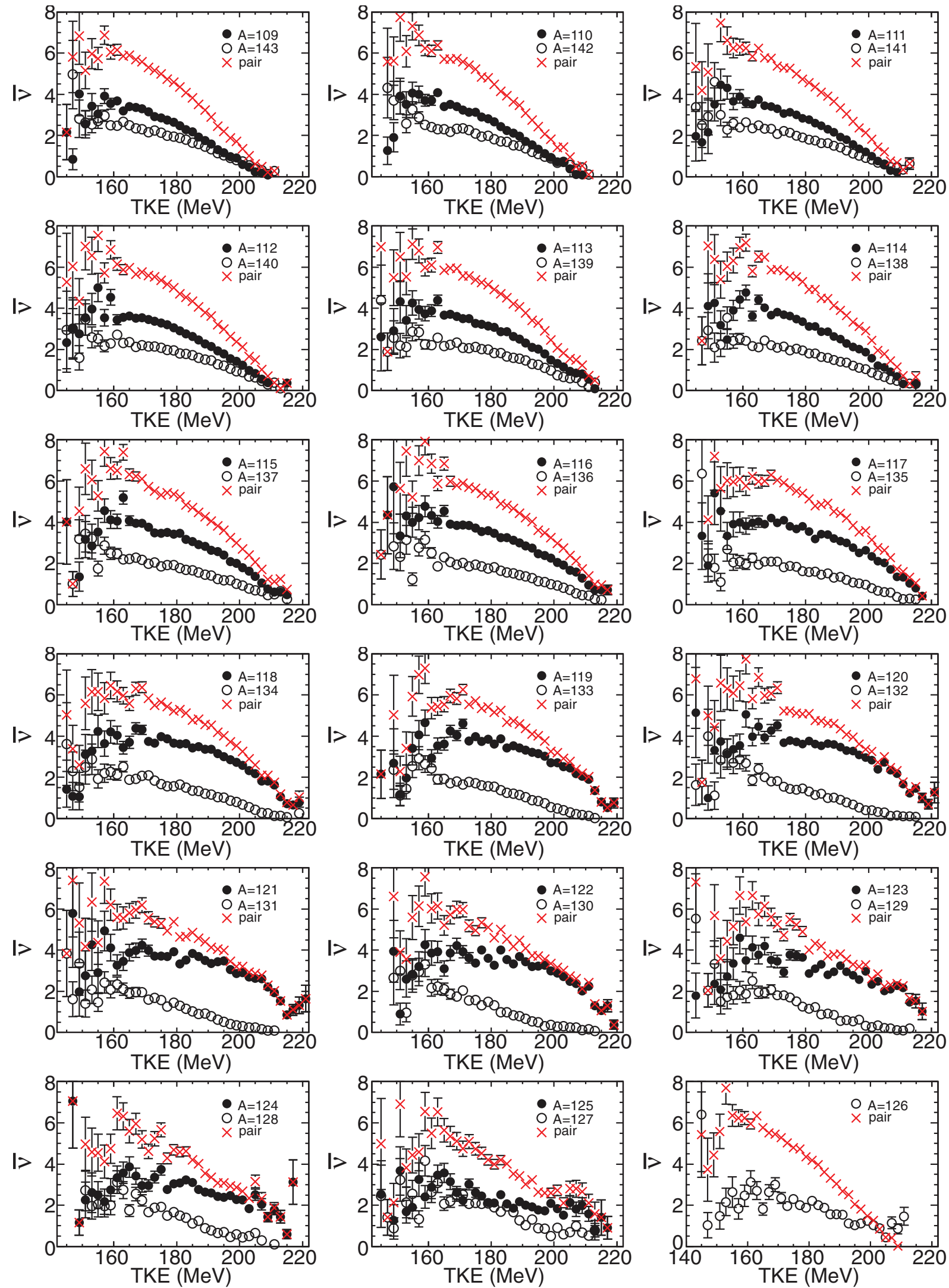

FIG. 13. (Color online) Dependence of the average number of neutrons emitted per light and heavy fission fragment (closed black and open white circles, respectively) as well as the average total neutron multiplicity of the pair of fragments (red crosses) as a function of TKE. 

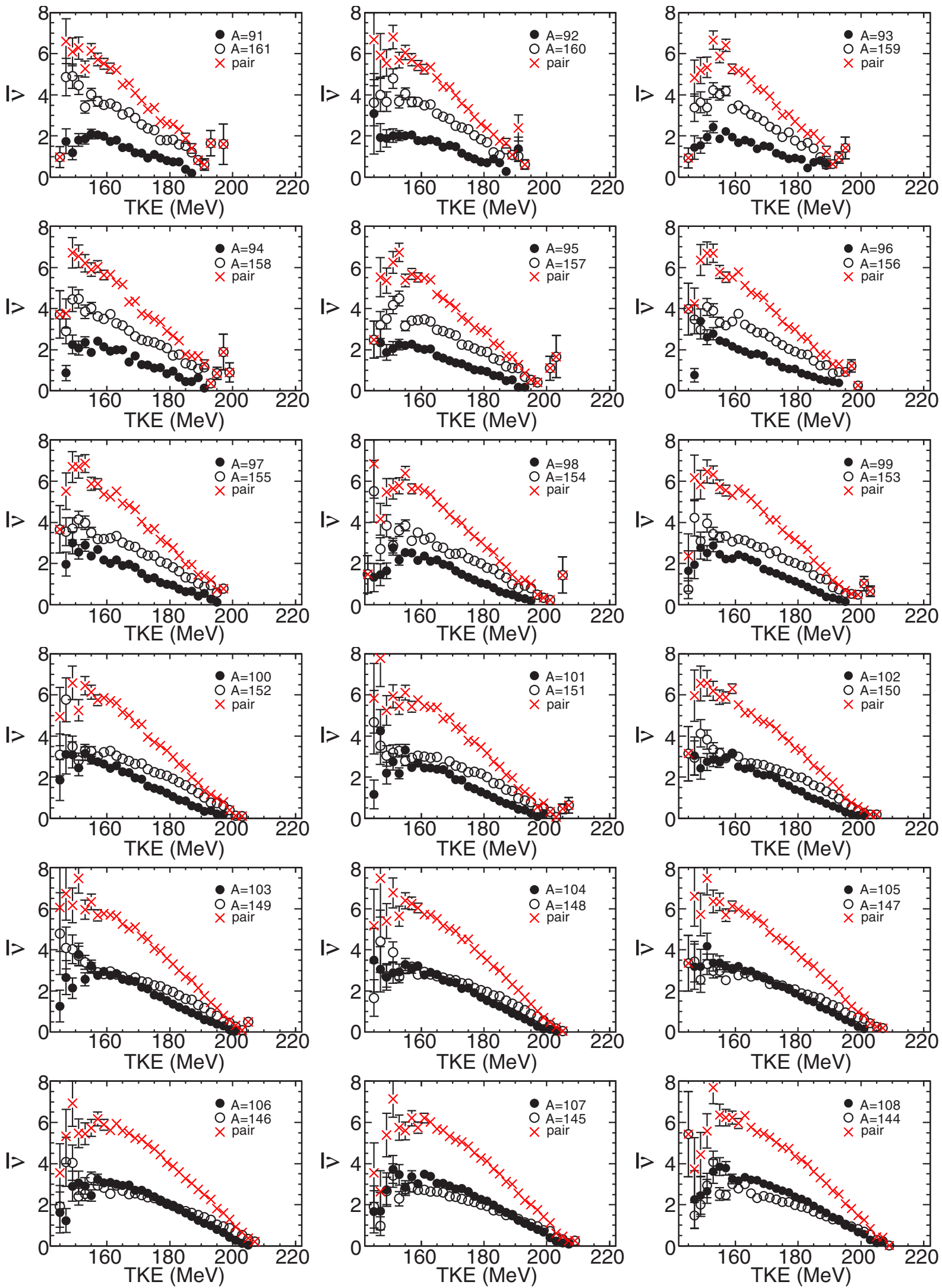

FIG. 14. (Color online) Dependence of the average number of neutrons emitted per light and heavy fission fragment (closed black and open white circles, respectively) as well as the average total neutron multiplicity of the pair of fragments (red crosses) as a function of TKE. 

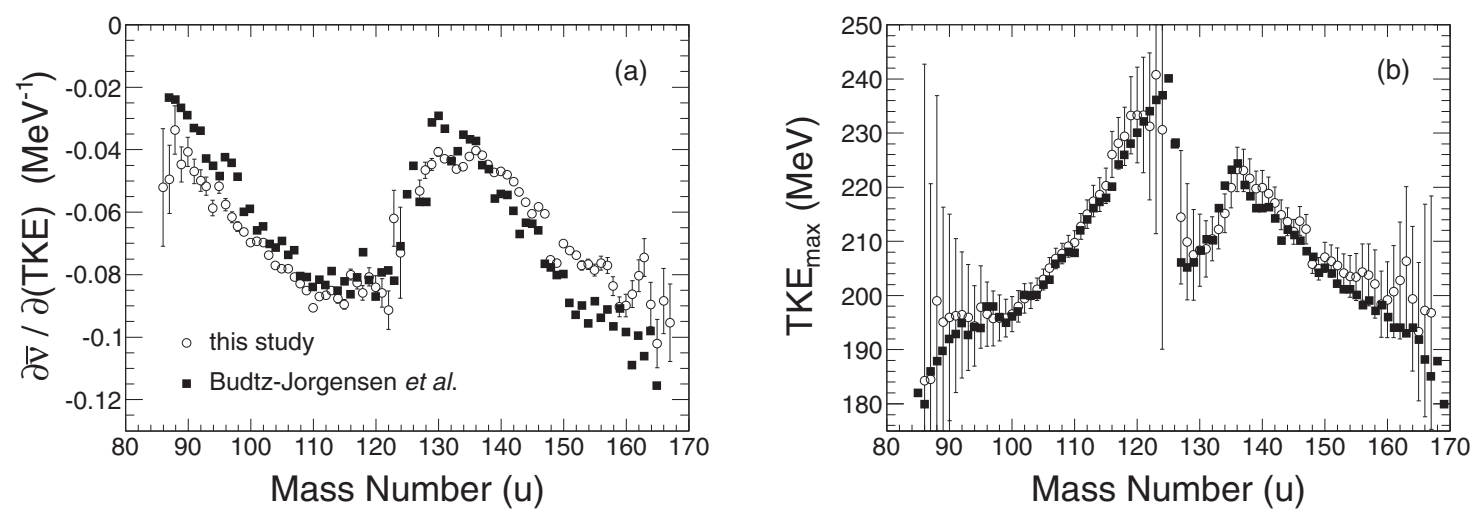

FIG. 15. (a) Slope of the neutron multiplicity per fragment with respect to the TKE as a function of fragment mass number. (b) TKE where the neutron emission stops as a function of fragment mass number. Data from Ref. [11] is included for comparison.

region between 85 and $100 \mathrm{u}$ observed in Refs. [22-25] is reproduced in this work. The reason it was not seen in Ref. [11] might be the obvious shift in mass numbers of the correction for the neutrons emitted from the backward-directed fragments in Ref. [11] (Fig. 8) compared with the present correction shown in Fig. 8. The present data confirm the increase in neutron multiplicity below mass number 82 , which indicates a third sawtooth, as observed in Ref. [11]. However, the present data do not show the expected complimentary decrease in neutron multiplicity for the far asymmetric heavy-fragment region. It has been shown in Ref. [15] that the fission-fragment yields at far asymmetric mass numbers $\left(m^{*} \lesssim 80\right)$, extracted from double-kinetic-energy experiments with large angular acceptance detectors, is greatly exaggerated due to misidentification of the fragment masses. This puts doubts also on the reliability of the neutron yields from fragments identified to have masses in this region. A reduction of the angular cone of accepted events to minimize the influence of multiple scattered fragments, as in Ref. [15], leaves only a few coincident events in the far asymmetric mass region. Hence, an experimental conclusion about the existence of a third sawtooth in the far asymmetric region is not possible from the present data set, nor from the data of Ref. [11].

In Fig. 11 the mean number of neutrons emitted per fission is shown as a function of the TKE of the fission fragments. Except at low TKE, the dependence is nearly linear. A least-squares fit to the data from the present experiment gives an inverse slope of $(12.6 \pm 0.2) \mathrm{MeV} / n$, which agrees well with two earlier experiments $[11,27]$ as well as with recent theoretical work [10]. This quantity must not be interpreted as the energy needed for a pair of fragments to emit one more neutron, as noted already by Ref. [27]. This is mainly because different TKE show different mass distributions. It is clear from Fig. 11 that the available experimental data show quite strong deviations, therefore effort was put into investigating this in detail. The first factor having a strong impact on the slope of $\bar{v}_{T}$ (TKE) is the correction for recoil energy imparted to the fission fragment by the detected neutron [19]. In an experiment covering a $4 \pi$ solid angle, the correction is theoretically nonexistent [27]; it is strongest for experiments where both neutrons and fission fragments are detected in a small solid angle. This explains the deviation of the historical data from Bowman et al. [26,28], where this was not considered. Because of the large solid angle subtended by the ionization chamber, the magnitude of the correction is in our case much smaller. The influence of this correction on $\bar{v}_{T}(\mathrm{TKE})$ in the present experiment is displayed in Fig. 12(a). A second effect that has a strong influence on $\bar{v}_{T}$ (TKE) is electronic instability. Evaluating the data from the stability monitor in this experiment, it was found that electronic temperature drifts on the order of a percent may be expected during the collection of fission-neutron coincidences. The time to collect enough statistics for the noncoincident distribution is, however, much shorter. Hence, if the coincident and noncoincident distributions are collected in separate runs, which is usually the case in order to reduce dead time, one may expect a difference in the calibration constant between the two cases. In Fig. 12(b) the red and blue lines represent the result obtained by artificially changing the energy calibration constant by $\pm 0.5 \%$ in the coincident events relative to the noncoincident events. The relatively big impact on the result of such a seemingly small systematic error can be understood by examining Eq. (7). The quantity $\bar{\nu}_{T}(\mathrm{TKE})$ results from the ratio of two nearly Gaussian distributions. Therefore, to estimate the impact of the electronic drift, which shifts one of these distributions by $0.5 \%(\sim 1 \mathrm{MeV})$, the shift should be compared with the width $(\sim 10 \mathrm{MeV})$ rather than the average $(\sim 180 \mathrm{MeV})$ of the distribution. A scenario closer to reality than a simple shift of the amplification is that the calibration constant for the coincident events scatter around that for the noncoincident events. The impact of this scenario on the experimental result was checked by randomly changing the calibration constant according to a Gaussian distribution, with a width of $1 \%$, in the analysis of the coincident events. The result is represented by black points in Fig. 12(b). At first glance one might have expected that randomly changing the calibration constant would average out the differences and that $\bar{v}_{T}$ (TKE) would be unaffected. This is, however, clearly not the case. In fact, $\bar{v}_{T}$ (TKE) is increased at low and high TKE, at the expense of $\bar{v}_{T}$ (TKE) in the region where the fission yield is the highest. This is because only one of the two measured distributions is smeared out. In conclusion, the result for $\bar{\nu}_{T}(\mathrm{TKE})$ is very sensitive to small systematic errors in the determination of the fission-fragment energies and great 


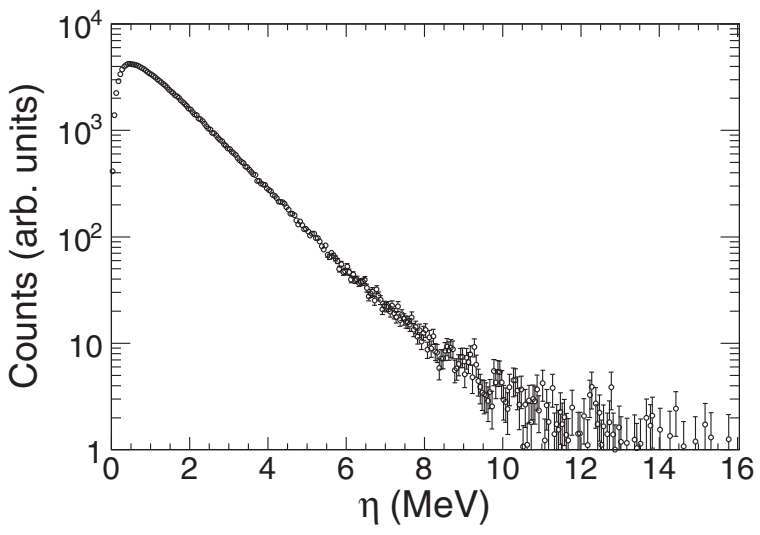

FIG. 16. Integral prompt-fission neutron spectrum in the fragment center-of-mass reference frame.

care must be exercised when obtaining and evaluating the experimental fission-fragment data.

\section{Fission-fragment mass and total-kinetic-energy correlations}

In Figs. 13 and 14 the dependencies on TKE of the average neutron multiplicity per fragment as well as the average total neutron multiplicity per fission, for fragment pairs with masses between 91 and 161, are shown. The variation with TKE is again nearly linear and is approximated by straight lines in the following. The slopes of the straight lines are related to the energy needed to emit one more neutron while the crossing point with the TKE axis is related to the $Q$ value of the fission. The average slope of the mean total multiplicity (represented by red crosses in Figs. 13 and 14) as a function of TKE corresponds to $7.3 \mathrm{MeV} / n$. This value compares well with the value $7.45 \mathrm{MeV}$ calculated from the sum of the average neutron binding energy (5.2 MeV [26]), the average center-of-mass neutron energy (1.45 MeV), and an additional $0.8 \mathrm{MeV}$ carried away by fission $\gamma$ rays [29].

For completeness, least-squares fitting to the average neutron multiplicity per fragment as a function of the TKE for individual masses were carried out and compared to the results

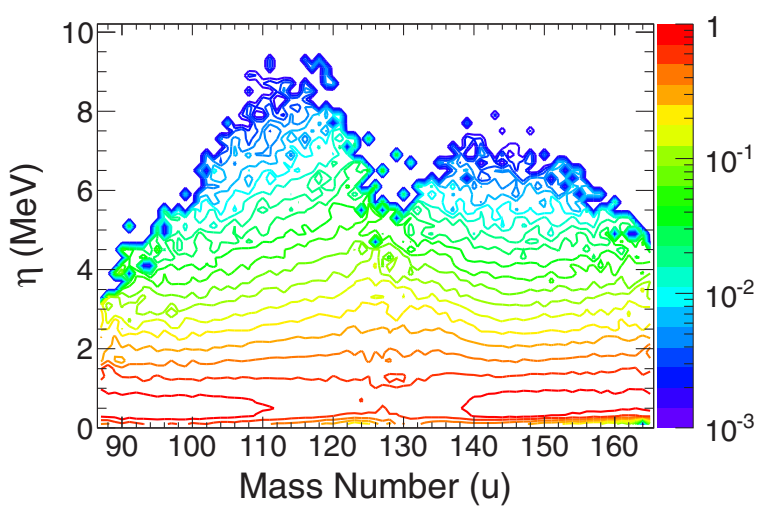

FIG. 17. (Color online) Contour plot of prompt-fission neutron spectra in the fragment center-of-mass reference frame as a function of the fragment mass number. The spectra are normalized to yield the same integral for each mass number.
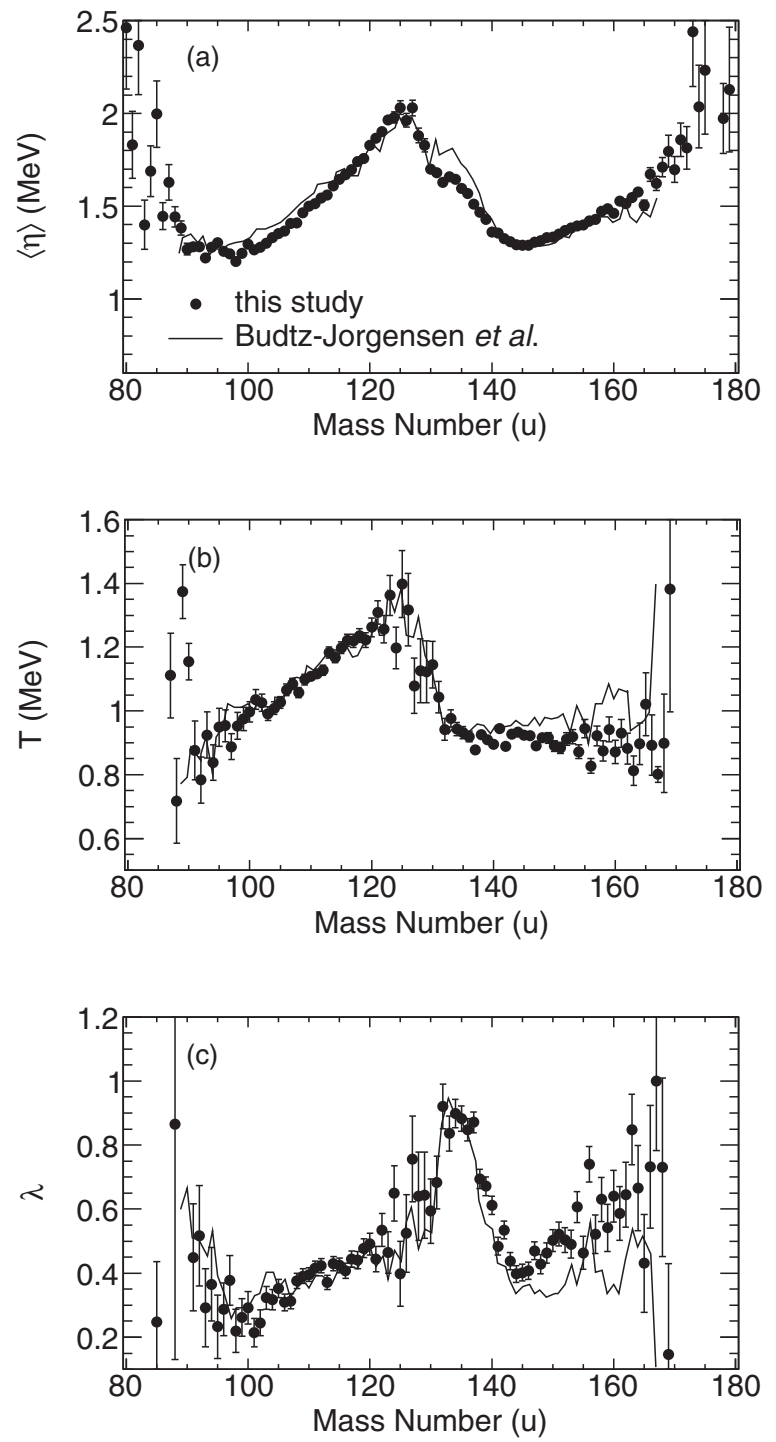

FIG. 18. (a) The average neutron energy in the fission-fragment center-of-mass reference frame, (b) nuclear temperature, and (c) cascade neutron emission coefficient as a function of fragment mass number.

from Ref. [11]. The results are obviously in good agreement, as can be seen in Fig. 15.

\section{Fission neutron spectrum in fragment rest frame}

The fission neutron spectrum in the rest frame of the fission fragment integrated over all masses and TKE is displayed in Fig. 16; the average neutron energy is $1.45 \mathrm{MeV}$. The spectrum has been fit by the cascade evaporation spectrum of Eq. (14). The obtained result of $T_{\text {eff }}=1.07$ and $\lambda=0.42$ is in fair agreement with the result of Ref. [11], who obtained $T_{\text {eff }}=1.07$ and $\lambda=0.38$. From the experimental data, the center-of-mass neutron-energy spectrum is available for each individual fission-fragment mass and TKE; a contour plot of the center-of-mass neutron energy as a function of the fragment mass number is displayed in Fig. 17. For comparison of the present result with those of Ref. [11], the spectra were 
evaluated by using Eq. (14); the result is displayed in Fig. 18, together with the average neutron energy in the center-of-mass frame. The earlier results from Ref. [11], represented by the full black line, are again confirmed by the present experiment.

\section{CONCLUSIONS}

A simultaneous investigation of prompt-fission neutrons and fission fragments in the spontaneous fission of ${ }^{252} \mathrm{Cf}$ has been performed as a preparatory step to the implementation of an array of neutron detectors to investigate prompt-fission neutron emission in resonance neutron-induced fission of ${ }^{235} \mathrm{U}$. Comparison of the results with data in the literature show that the experimental setup and analysis is under control. Open questions involving neutron emission in spontaneous fission of ${ }^{252} \mathrm{Cf}$ have been addressed.
The apparent increase in neutron yield as a function of fission-fragment mass, $\bar{v}(A)$, for far asymmetric fission observed in Ref. [11] is confirmed. However, the far asymmetric region is heavily influenced by energy degradation of the fragments and therefore we do not consider this as proof of two new saw teeth as conclude by Ref. [11]. Such a conclusion would need a reduced angular acceptance, to minimize the influence of energy degradation, and at least a two order of magnitude increase in counting statistics.

Particular attention was paid to the total neutron multiplicity dependence on the TKE of the fission fragments. An inverse slope of $(12.6 \pm 0.2) \mathrm{MeV} / n$ is obtained from the present investigation. The main factor affecting this dependence is the treatment of the recoil energy imparted on the fission fragments detected in coincidence with a neutron, as already realized by Gavron [19]. A second factor identified to affect this dependence is electronic instability.
[1] F.-J. Hambsch, H. H. Knitter, C. Budtz-Jørgensen, and J. P. Theobald, Nucl. Phys. A 491, 56 (1989).

[2] F.-J. Hambsch, I. Ruskov, and L. Dematté, in Proc. Scientific Workshop on Nuclear Fission Dynamics and the Emission of Prompt Neutrons and Gamma Rays (Theory-1), EUR 24802 (Sinaia, Romania, 2011), p. 41.

[3] R. E. Howe, T. W. Phillips, and C. D. Bowman, Phys. Rev. C 13, 195 (1976).

[4] A. Al-Adili, F.-J. Hambsch, S. Pomp, and S. Oberstedt, Phys. Rev. C 86, 054601 (2012).

[5] A. Tudora, Ann. Nucl. Energy 53, 507 (2013).

[6] S. Zeynalov, F.-J. Hambsch, and S. Oberstedt, J. Korean Phys. Soc. 59, 1396 (2011).

[7] D. Regnier, O. Litaize, and O. Serot, Nucl. Sci. Eng. (La Grange Park, IL, US) 174, 103 (2013).

[8] S. Lemaire, P. Talou, T. Kawano, M. B. Chadwick, and D. G. Madland, Phys. Rev. C 72, 024601 (2005).

[9] B. Becker, P. Talou, T. Kawano, Y. Danon, and I. Stetcu, Phys. Rev. C 87, 014617 (2013).

[10] O. Litaize and O. Serot, Phys. Rev. C 82, 054616 (2010).

[11] C. Budtz-Jorgensen and H.-H. Knitter, Nucl. Phys. A 490, 307 (1988).

[12] A. Al-Adili, F.-J. Hambsch, S. Oberstedt, S. Pomp, and S. Zeynalov, Nucl. Instrum. Methods Phys. Res., Sect. A 624, 684 (2010).

[13] W. Mannhart, in Proceedings of an Advisory Group Meeting on Properties of Neutron Sources Organized by the International Atomic Energy Agency and Held in Leningrad, USSR, 9-13 June 1986 (IAEA, Vienna, 1987), p. 158.
[14] N. Kornilov, I. Fabry, S. Oberstedt, and F.-J. Hambsch, Nucl. Instrum. Methods Phys. Res., Sect. A 599, 226 (2009).

[15] F.-J. Hambsch and S. Oberstedt, Nucl. Phys. A 617, 347 (1997).

[16] A. Göök, F.-J. Hambsch, A. Oberstedt, and S. Oberstedt, Nucl. Instrum. Methods Phys. Res., Sect. A 664, 289 (2012).

[17] C. Budtz-Jørgensen, H.-H. Knitter, C. Straede, F.-J. Hambsch, and R. Vogt, Nucl. Instrum. Methods Phys. Res., Sect. A 258, 209 (1987).

[18] F.-J. Hambsch, J. Van Aarle, and R. Vogt, Nucl. Instrum. Methods Phys. Res., Sect. A 361, 257 (1995).

[19] A. Gavron, Nucl. Instrum. Methods 115, 99 (1974).

[20] K. Le Couteur and D. Lang, Nucl. Phys. 13, 32 (1959).

[21] M. Chadwick et al., Nucl. Data Sheets 112, 2887 (2011).

[22] V. Dushin et al., Nucl. Instrum. Methods Phys. Res., Sect. A 516, 539 (2004).

[23] R. Walsh and J. Boldeman, Nucl. Phys. A 276, 189 (1977).

[24] V. Zakharova and D. Ryazanov, Sov. J. Nucl. Phys. 30, 19 (1979).

[25] C. Signarbieux, J. Poitou, M. Ribarg, and J. Matuszek, Phys. Lett. B 39, 503 (1972).

[26] H. R. Bowman, J. C. D. Milton, S. G. Thompson, and W. J. Swiatecki, Phys. Rev. 129, 2133 (1963).

[27] H. Nifenecker, C. Signarbieux, R. Babinet, and J. Poitou, in Proc. Third Symposium on Physics and Chemistry of Fission (IAEA, Vienna, 1974), Vol. 2, p. 51.

[28] H. R. Bowman, S. G. Thompson, J. C. D. Milton, and W. J. Swiatecki, Phys. Rev. 126, 2120 (1962).

[29] R. Billnert, F.-J. Hambsch, A. Oberstedt, and S. Oberstedt, Phys. Rev. C 87, 024601 (2013). 